\title{
PROPER TIME IN TECHNICAL SCIENCES AND ALTERNATIVE NOTIONS FOR THE FORCE, MECHANICAL WORK AND HEAT
}

\author{
ALEXEI MAFTULEAC ${ }^{*}$ \\ ${ }^{1}$ Institute of Chemistry, Academy of Sciences of Moldova, Academiei str. No. 3, Chișinău, \\ MD-2028, Republic of Moldova
}

\begin{abstract}
The notion of proper (localized) time is one of the more important in the theory of relativity and is often used to explain phenomena that occur in conditions of high speeds or gravitation. In this paper, on the basis of the concept of proper (localized) time we tried to explain the functioning of simple mechanisms as a change in their space/time relationships, and to consider from another point of view some notions of physics (mechanics). A hypothesis about space and time equivalence and their mutual compensating change in every phenomenon that occurs, has been proposed. This hypothesis is substantiated with formulas and data from literature. This article may be of interest to the physicists and engineers.
\end{abstract}

Keywords: simple machines, proper (localized) time, space-time relationships of mechanisms, alternative notions for physical sizes.

\section{INTRODUCTION}

The greatness of human intellect consists in the fact that the man understood causal links between natural phenomena and began to use them in his interests. From the most ancient times until now we use the simple machines, their modifications and combinations [1-4]. The principle underlying the action of these ingenious mechanisms is the Golden Rule of Mechanics which has been understood since school. We, using simple machines do not win in mechanical work (energy), but in force only, losing in the distance [5]. We usually do not analyze what happens in such a way. Thus, the Golden Rule of mechanics turned into postulate. However, we forget that the machines work in the time. But what is the time? According to philosophers [6], time is an order of events that occur in space. Majority of physicists usually avoid giving answer to this question, but there are a lot of opinions on the nature of time, different aspects of its size [7-10] including some that deny reality of time, considering it a mere mathematical dimension [9]. The question of the nature of time is one of the most difficult and beyond the scope of this article; nevertheless in no way we could not pass it around. The notion of Proper Time came for the first time in Special Theory of Relativity [11-13], but Einstein gave no explicit definition of this physical size. In this paper here is used the notion "proper time" of mechanism which is in interaction with the environment, videlicet, having an energetic state.

\section{THE AIM OF THE STUDY AND ITS THEORETICAL FOUNDATIONS}

The purpose of this work was an analysis of the well-known laws of mechanics and to show applicability to them of modern concepts of proper, or localized time. At the same time, we wanted to show the difficulty of understanding what the Time is and everything connected with it. Publications on simple machines, their combinations and properties [1-4] and modern concepts of time and space-time relationships served as a theoretical

\footnotetext{
*Corresponding author, emails: alexei.maftuleac@yahoo.com, amaft@mail.md

(C) 2016 Alma Mater Publishing House
} 
basis [11-17] for the paper. The basic concept of the article is the idea that space and time are the physical sizes complementary one to another, and everything that exists is subject to the laws of fractality and by analyzing the simple things we can get to understand more complicated things and phenomena. That is why in the beginning was analyzed the properties of the lever - the simplest of the mechanisms.

\section{RESULTS AND DISCUSSIONS}

\subsection{The simple machine. Lever and the Law of the Lever}

The simple machine is an installation in which the work can be rendered by the mechanical energy only. So the simple machines (mechanisms) such as lever, inclined plane, pulley and others $[1,2]$ serve to change the force.

Let's analyze the lever - the simplest mechanism that shows a solid body (a rigid bar) which can be rotated around a fixed point called a fulcrum. Lever action principle is a direct consequence of the Law of Energy Conservation, according to which, in a closed system the work of the action and reaction forces are equal [2]:

$$
F_{1} \Delta h_{1}=F_{2} \Delta h_{2}
$$

As the displacement of the lever arms is proportional to their length, we have:

$$
\frac{\Delta h_{1}}{\Delta h_{2}}=\frac{L_{1}}{L_{2}} \text { and } \quad F_{1} L_{1}=F_{2} L_{2}
$$

Of course, equality (2) is valid for ideal lever only, videlicet, in the absence of friction force.

Let's put the lever horizontally (in the same gravitational conditions, Figure 1) in a rotation movement with the axis passing through the fulcrum.

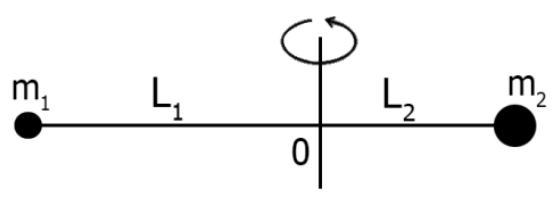

Fig. 1. Lever that rotating in a horizontal plane.

In the case of a constant angular speed, the linear, or circumferential speed of bodies $\mathrm{m}_{1}$ and $\mathrm{m}_{2}$ will be the following:

$$
V_{1}=\frac{2 \pi L_{1}}{\tau_{1}} \quad \text { and } \quad V_{2}=\frac{2 \pi L_{2}}{\tau_{2}}
$$

where $L_{1}$ and $L_{2}$ are length of the arms, or rays of circumferences described by the bodies, $\tau_{1}$ and $\tau_{2}$, respectively, their proper time. It should be mentioned that each of the rotating bodies are different values of proper time, and these values are different from the value of time measured by their rotation angle described by the lever arms. Such situation is due to different spatial conditions of the bodies and of the observer. This follows from Einstein's theory of relativity $[12,13]$ and was mentioned in [14-16]. Of course, this difference of time that we have in discussion is very small and it has mostly only a theoretical significance. In a compressed or small space this physical size (the time) can be more significant.

\subsection{The Law of Conservation of Momentum}

Momentum, or the Amount of Movement of a body, by definition, is the result of multiplying the mass and velocity $[17,18]$ :

$$
P=m V
$$

If the sum of all external forces acting on the body is equals zero, the vector of Momentum has constant module and direction [19]. 


$$
P=\sum_{i=1}^{n} P_{i}=\sum_{i=1}^{n} m_{i} V_{i}
$$

Referring to lever in rotation (Figure 1), formula (5) can be represented as follows:

$$
m_{1} V_{1}=m_{2} V_{2} \quad \text { or } \quad \frac{2 \pi L_{1}}{\tau_{1}} m_{1}=\frac{2 \pi L_{2}}{\tau_{2}} m_{2}
$$

From equality (6) we have:

$$
m_{1} L_{1} \tau_{2}=m_{2} L_{2} \tau_{1}
$$

where $L_{1}$ and $L_{2}$ are radiuses, or lever arms, $\tau_{1}$ and $\tau_{2}$ are respective proper time of the bodies $m_{1}$ and $m_{2}$ in rotating movement.

If the lever does not rotate, the proper time $\tau_{1}$ and $\tau_{2}$ are equal values (depending on local gravitational acceleration) and the formula (7) becomes simpler:

$$
m_{1} L_{1}=m_{2} L_{2}
$$

Multiplying both sides of the equality (8) to gravitational (or centrifugal) acceleration we can obtain a formula identical to formula (2). Thus we can say that formula (7) is a generalized Law of the Lever including the values of proper time. By the way, the Law of Conservation of Momentum is due to the homogeneity of the Space [17].

\subsection{The Law of Conservation of Energy}

During rotation, each of the bodies (Figure1) on the lever has a certain amount of kinetic energy, and if the lever arms are balanced, energies of bodies are equal:

$$
\frac{1}{2} m_{1} V_{1}^{2}=\frac{1}{2} m_{2} V_{2}^{2}
$$

where $V_{l}$ and $V_{2}$ are the linear speeds of the bodies $m_{1}$ and $m_{2}$. Replacing in (9) the value of the speed

$$
V=\frac{L}{\tau}
$$

and simplifying coefficients, we obtain:

$$
m_{1} \frac{L_{1}^{2}}{\tau_{1}^{2}}=m_{2} \frac{L_{2}^{2}}{\tau_{2}^{2}}
$$

and:

$$
m_{2} L_{1}^{2} \tau_{2}^{2}=m_{2} L_{2}^{2} \tau_{1}^{2}
$$

Each of the lever arms during movement describes circular sectors with the same central and opposite angle, but with different areas, due to different rays. Multiplying both sides of equality (12) to $\frac{\pi n}{360}$ or $\frac{\alpha}{2}$, where these fractions are the sizes of central angle in degrees or radians, we obtain another expression of the Generalized Lever Law:

$$
m_{1} S_{1} \tau_{2}^{2}=m_{2} S_{2} \tau_{1}^{2}
$$

where $S_{1}$ and $S_{2}$ are the areas of the sectors. As seen from the above, and here (13) the values of proper time of the bodies are presented.

It should be mentioned here that the Law of Energy Conservation is due to homogeneity of the Time [17]. Homogeneity of Space and Time, however, implies that systems exist in extended astronomical sizes. In localized systems where there are massive bodies and strong physical fields, it is expected to be local Space and Time inhomogeneous. The theory about this heterogeneity with theoretical arguments is described in [20]. 


\subsection{The using of the notion of proper time in technical sciences}

It is known that many of scientific categories (concepts) used currently have been introduced a long time ago - a few hundred or even 1-2 millennia ago. Over time, in the result of the development of our knowledge, these concepts have been fortified and their use was extended.

Others, on the contrary, have been radically changed. The evaluation of the concepts continues today. As an example, such concepts as inertia, centrifugal force and the centripetal one and many others are under discussion. Some experts admit their real existence, others - no, considering them fictive, artificially introduced just for to explain something. This interesting and complex question in mechanics leads to heated debates among experts. As an example regarding this matter there serve the reasoning and arguments in such debates of the famous scientist, specialist in theoretical mechanics - Astakhov [21]. He defends the thesis of the real existence of inertia and other similar forces.

Among other, the study of circular movement will help to find the way to understanding the phenomenon of Time. In our opinion the Time does not exist without the Space and outside it. In the studies of rotational movement these both concepts (Space and Time) seem simple, and at the same time interconnected. Based on the above, we will try to explain, for example, the appearance of force, and what is the difference between heat and mechanical work.

Suppose we have a lever in rotation and one of the lever arms is in role of circumference radius. The radius is divided into smaller segments equal in length (Figure 2). These segments during rotation around the center, describe corresponding arches and areas in the plane of rotation. (Rotation occurs in horizontal plane, in homogeneous gravitational conditions). Although the length of the radius segments is one and the same, described arches and areas are different. In result, their spatial-temporal conditions are different (we consider that their mass is not changed). According to Einstein's theory $[12,13]$, we can say that these segments are in places with different curvatures of space-time. But this theory is not simple to use because of complicated mathematics. Also this theory is better suited for extreme cases - high speed and strong gravity. For normal conditions it is much easier to use the concept and notion of proper (localized) time.

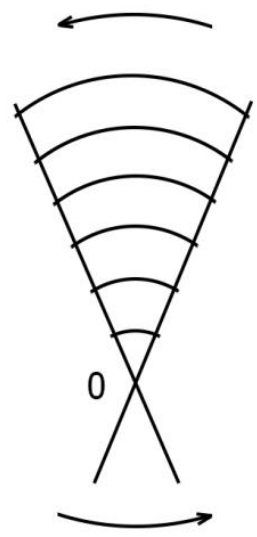

Fig. 2. Divided lever arm in rotation movement.

\subsection{Emergence of the centrifugal force}

Material points or segments on lever arms, with different distance from the center, have different values of their proper time during rotation movement. Likewise, linear speed on the circumference and the amount of their Momentum will be different, depending on the distance from the center of rotation. Let us take the expression (4) differentiating it in such a way:

$$
\frac{d P}{d \tau}=\frac{d(m V)}{d \tau}=m \frac{d V}{d \tau}
$$

where $P$ is momentum, $\tau$ - proper (localized) time, $V$ - linear speed of rotating body, and size $d V / d t$ is acceleration. As the rotating speed is low ( $V \ll c$, where $c$ is speed of light), the effect of mass changing can be ignored. 
We get the expression for the centrifugal force from (14):

$$
\frac{d P}{d \tau}=m \frac{d V}{d \tau}=m a=F_{c}
$$

In this way, using the notion of proper time, or more correctly, using difference in space/time localization of the segments (parts) of solid body in rotation, we can explain the emergence of centrifugal force during rotation movement and that this force is not artificially introduced, but really exists.

\section{EXPANSIVE PHENOMENA IN SPACE - RESTRICTED IN TIME, AND VICE VERSA}

Let's go back to the Gold Law of Mechanics. Why, however, using simple machines, we do not gain in energy, but only in force, and what do we change in this case? As seen from the equality (11), the value of energy depends proportionally on the mass and the square of the spatial size, and inversely is proportional to the square of the temporal one. Here's the answer - by using mechanisms, we change the values of the sizes that fall in the expression of energy, but with mutual compensation, therefore does not change the value of energy. Let's analyze in more detail the expression of energy. As already indicated above in equality (11), the energy value is expressed as follows:

$$
E=\frac{m L^{2}}{\tau^{2}}
$$

Differentiating this expression, and after simplifying it, we get the following result:

$$
d E=d\left(\frac{m L^{2}}{\tau^{2}}\right)=\frac{L^{2}}{\tau^{2}} d m+\frac{2 m L}{\tau^{2}} d L-\frac{2 m L^{2}}{\tau^{3}} d \tau
$$

Each of the physical quantities ( $m, L$, and $\tau$ ) may undergo changes monotonous in their states ranges 1 and 2 . Then, integrating obtained differential (17), we will have:

$$
\int_{E_{1}}^{E_{2}} d E=\frac{L^{2}}{\tau^{2}} \int_{m_{1}}^{m_{2}} d m+\frac{2 m}{\tau^{2}} \int_{L_{1}}^{L_{2}} L d L-2 m L^{2} \int_{\tau_{1}}^{\tau_{2}} \frac{d \tau}{\tau^{3}}
$$

and

$$
\Delta E=E_{2}-E_{1}=\frac{L^{2}}{\tau^{2}}\left(m_{2}-m_{1}\right)+\frac{m}{\tau^{2}}\left(L_{2}^{2}-L_{1}^{2}\right)+m L^{2}\left(\frac{1}{\tau_{2}^{2}}-\frac{1}{\tau_{1}^{2}}\right)
$$

According to the above mentioned Golden Rule of Mechanics, using lever (or other simple mechanism), we do not win in work (energy), thus the result from integration (19) can be considered by us equal to zero:

$$
\frac{L^{2}}{\tau^{2}}\left(m_{2}-m_{1}\right)+\frac{m}{\tau^{2}}\left(L_{2}^{2}-L_{1}^{2}\right)+m L^{2}\left(\frac{1}{\tau_{2}^{2}}-\frac{1}{\tau_{1}^{2}}\right)=0
$$

Considering $\Delta m=m_{2}-m_{1}=O$ (mass remains constant), we omit in equation (20) the first term and we get:

$$
\frac{m}{\tau^{2}}\left(L_{2}^{2}-L_{1}^{2}\right)+m L^{2}\left(\frac{1}{\tau_{2}^{2}}-\frac{1}{\tau_{1}^{2}}\right)=0
$$

By distributing in both sides of the equality (21) the remaining terms and by simplifying the value of $m$, we get:

$$
\frac{1}{\tau^{2}}\left(L_{2}^{2}-L_{1}^{2}\right)=-L^{2}\left(\frac{1}{\tau_{2}^{2}}-\frac{1}{\tau_{1}^{2}}\right)
$$

We observe that the left side of the equality (22) represents a spatial change at the constant value of time (more correctly, to very small changes of time), and the right - a temporal change that have a constant spatial size (or very small changes). Also from here, we can make the following conclusion: any spatial change inevitably leads to a respective equivalent of temporal change, in compensating way. Any phenomenon that happens in nature occurs expansively in space and restricted in time, or vice versa, expansively in time and restricted in space. With a certain dose of risk (we can be wrong), using the equation (22), we can give definition of Time. In our opinion, 
the Time is an altered state of Space, or Space in changing, with an excitation state, so the intensity of any process is a measure of the disturbance of the space. One thing is clear, that real, physical time is not that measure the clock, the time is associated with the energy state of the body (or system). Possible, such a state can have a space with great energy density. Space and Time are the complementary quantities, as two parts of a one whole, when one part increases, the other one decreases. The idea of time and space equivalence is not something new; it has been submitted by other researchers [22]. According to the conception exposed in [23], the difference between time and space consists in their asymmetry. But the question about symmetry or asymmetry of these sizes seems to be still under discussion. The relationship between space and time can be played by the expression that reminds Pythagorean Theorem:

$$
\tau^{2}+\left(-s^{2}\right)=i^{2}
$$

where $\tau=c t(24)$ is the temporal size ( $\tau$ is proper time of moving body, $\mathrm{t}$ - the time in the motionless reference system and $c$ - speed of light), $s-$ the spatial size, and $i=\sqrt{-1}$.

Dividing the equality (23) to $c^{2} t^{2}$ we get the following relationship:

$$
1-\frac{v^{2}}{c^{2}}=-\frac{1}{c^{2} t^{2}}
$$

And what is then the Space? The Space is the unlimited great three-dimensional realm in which all material objects are located and all events occur [24]. There is another definition for Space [25] - it is boundless extent in which objects and events have relative position and direction. Modern concepts of physicists-theorists give such response: the Space is a form of the Quantum Vacuum [26, 27]. Is this a return to the theory of the ether, but on another level? It is difficult to answer. But let us return to the notion of a proper (localized) time. What does the practical use of this concept give in technical sciences? It allows looking and understanding otherwise the known phenomena and objects studied, for example, by the static mechanics, aero - and hydrodynamics etc. This is useful for physicists and engineers - teachers, students and inventors because it helps to develop the imagination and see things and events from a different angle. The Force can be defined as a physical factor that hinders the change in the spatial/temporal relationship of the body, or is the response of it to this change. Work is the physical quantity that defines the changing that occurs predominantly in the Space, and respectively, Heat - the changing that occurs predominantly in the Time. For comparison, let us remind here that in thermodynamics there are two types of heat capacity for conditions with constant pressure or constant volume [28]:

$$
\left(\frac{\partial H}{\partial T}\right)=C_{p} \text { and }\left(\frac{\partial U}{\partial T}\right)=C_{v},
$$

where $H$ is enthalpy, $U$-internal energy and $T$ - temperature.

Perhaps this is evidence of the correctness of such a concept as described above. One of these thermodynamic processes are carried out by mechanical work performance (predominantly expansive in Space), another - by temperature change in the system (predominantly expansive in Time). Also, it can be said that Energy of a system is the size characterizing potential (possibility, capacity) of this system to do work or to emit (or absorb) heat. Location of the system in space-time continuum depends on its energy. In this regard, Energy appears as analog of space-time curvature in Einstein's theory of relativity. Changing energetic state of a body (or system) from a value to another one is equivalent to changing the value of curvature of that part of the space-time in which the body (system) is. Such conception, seems, not to contradict the conception of specialists in theoretical physics [29] operating with density of Quantum Vacuum. Possibly discussions on this theme strengthens foundation of a new branch of science, called Chronodynamics, or Thermodynamics of the Time. (Here it mustn't be confused with another science - chromodynamics). The scope of application of such science can be the studying of processes with displacement in the time or/and with uneven passing (flow) of time. We remind you that in this paper the Time is regarded as excited state of the Space (and/or measure of this state), therefore in this sense we can assume that time travel (a sort of passage from one state to another) is possible, but this it was said in the context of a probability. Chronodynamics as a science generally accepted already does not exist, but there are many articles in which authors show the results of theoretical research related to space-time relationships of microcosm [30, 31] and conclusions of which coincide with the ideas underlying the chronodynamics. But what is common between lever or other simple machines and chronodynamics? Just on the basis of the simplest and well known things and phenomena, and accepting the thesis of the fractality of everything (of all that exist), including even the entire Universe $[32,33]$, we can understand something more complex and rise to a higher level of knowledge. 


\section{CONCLUSIONS}

Theoretical basis of mechanics was developed a long time ago and was the result of insights gained from the practice generalization. Accelerated development of technical sciences insists on adapting basic laws of mechanics to modern concepts about space and time, since a mechanical device is also part of space-time continuum, moreover the active its part.

Any mechanical installation works in space and time, so it is important to understand the notions of space and time, the interdependence of these sizes to clarify the basic principle of operation of the mechanism. This is useful for every physicist and engineer - teacher, student or inventor. An exclusive importance this will have to understand the operation of micro- and nano-mechanisms. When diminish the space, the time becomes more and more important.

Any spatial change inevitably leads to a respective equivalent change in the time and any phenomenon that happens in nature occurs expansively in space and restricted in time, or vice versa. Where exist a movement, the development, there is a change of the space/time relationships.

The new domains of research recently appeared and the ones that will emerge in the future insist to review and complete existing scientific notions and concepts. Only a deeper understanding of spatial/temporal relationships and accepting concept of fractality of all things and phenomena will allow us to climb to a higher level of knowledge.

\section{REFERENCES}

[1] https://en.wikipedia.org/wiki/Simple_machine. (10.09.2016).

[2] https://en.wikipedia.org/wiki/Lever (23.09.2016).

[3] Artabolevskij, I.I., Mekhanizmy v sovremennoj tekhnike. Tom 1. Elementy mekhanizmov. Prostejshie rychazhnye i sharnirno-rychazhnye mekhanizmy, Moskva, Nauka, 1979.

[4] Uicker, J., Pennock, G., Shigley, J.E., Theory of Machines and Mechanisms, 4th edition, Oxford University Press, 2010

[5] Kostrykin, M.I. ,Osnovy tekhnicheskoj mekhaniki, Izdanie 2, Moskva, Mashinostroenie, 1973.

[6] https://www.iep.utm.edu/time/ (04.10.2016).

[7] https://en.wikipedia.org/wiki/Time (04.10.2016).

[8] https://www.exactlywhatistime.com (04.10.2016).

[9] Fiscaletti, D., Sorli, A., Perspective about Quantum Mechanism in a Model of a Tree-Dimensional Quantum Vacuum where Time is a Mathematical Dimension, Sop Transaction on Theoretical Physics, vol.1, no. 3, 2014, p. 2372-2487.

[10] Mageshwaran, M., Exact Solution for Time and Entanglement in UDE Cosmology, American Journal of Modern Physics, vol. 5, 2016, p.14-19.

[11] https://www.iep.utm.edu/proper-t/ (13.10.2016).

[12] Rindler, W., Introduction to Special Relativity, 2nd edition, Oxford University Press, 1991.

[13] Hobson, M., Lasenby, N., General Relativity. An introduction for physics. (The equivalence principle and space-time curvature). Cambridge University Press, 2006, p. 147-175.

[14] Maftuleac, A., Space-time relationships and thermal effects in centrifugal field, International Journal of Modern Physics and Applications, vol. 1, no. 2, 2015, p. 17-21.

[15] Chugreev, Yu.V., Special relativity theory and experiments in centrifuge, Serpuhov, Inst. for High Energy Physics. Preprint, 1987.

[16] Chugreev, Yu.V., Relyativistskie effekty vo vrashhayushhejsya sistemy otscheta, Fizika elementarnykh chastits i atomnogo yadra, vol. 20, no. 3, 1989, p.669-693.

[17] Matveev, A.N., Mekhanika i teoria otnositelnosti, Moskva, ONICS 21 vek: Mir i obrazovanie, 2003.

[18] Astakhov, A.V., Kurs fiziki. Tom 1. Mekhanika. Kineticheskaya teoria materii, Moskva, Izdatelstvo fizicomatematichescoy literatury, 1977.

[19] Targ, S.M., Theoretical mechanics: a short course, Moscow, Mir (in Eng., transl. from Russian), 1976.

[20] Bogoslavskiy, G.Yu., Teoria localnogo anizotropnogo prostranstva-vremeni, Moskva, Izdatelstvo MGU, 1992, p. 256-264.

[21] https://www.sciteclibrary.ru/texts/rus/stat/st5378.pdf. (16.08.2016). 
[22] Kudukula, S.P., Space Time Equivalence - A new Concept, International Journal of Scientific and Research Publications, vol.2, no. 10., 2012, p. 1-2.

[23] http://rspa.royalsocietypublishing.org/content/royprsa/472/2185/20150670.full.pdf (30.08.2016)

[24] https://www.dictionary.com/briwse/space (30.08.2016).

[25] https://en.wikipedia.org/wiki/Space. (30.08.2016).

[26] Fiscaletti D. and Sorli A., Dynamic Quantum Vacuum and Relativity, Annales Universitatis Mariae CurieSkłodowska, Liublin-Polonia, Sectio AAA (accepted for publication), 2016.

[27] Burdyuzha, V., Vereshkov, G., Cosmology of Vacuum, Astrophys. Space Science, vol. 305, no. 3, 2006, p.235-239.

[28] https://en.wikipedia.org/wiki/Heat_capacity. (13.10.2016).

[29] Fiscaletti, D., Sorli, A., Space-Time Curvature of General Relativity and Energy Density of a TreeDimensional Quantum Vacuum, Annales Universitatis Mariae Curie-Skłodowska Lublin-Polonia, vol. 65, 2014, p. 53-77.

[30] https://arXiv.org/pdf/hep-th/0212232.pdf. (31.01.2017).

[31] http://vanikaghajanyan.narod.ru/vanik5.pdf (13.10.2016)

[32] Mandelbrot, B.B., The fractal geometry of Nature, New York, W.H. Freeman and company, 1983.

[33] King, C., Why the Universe is Fractal, Prespacetime Journal, vol. 3, No 3, 2012, p. 1-10. 